\title{
On the estimation of absolute grating groove density and inter-grating groove density errors of laser pulse compression gratings
}

\author{
A K SHARMA ${ }^{1,2, *}$ and A S JOSHI ${ }^{1}$ \\ ${ }^{1}$ Advanced Lasers and Optics Division, Raja Ramanna Centre for Advanced Technology, Indore 452013, India \\ ${ }^{2}$ Homi Bhabha National Institute, Mumbai 400094, India \\ e-mail: aksharma@rrcat.gov.in
}

MS received 13 March 2018; accepted 27 September 2018; published online 9 February 2019

\begin{abstract}
In this paper, experimental studies on estimation of absolute groove density of gratings and intergrating groove density errors are reported with typical detector limited accuracies of \pm 0.23 lines $\mathrm{mm}^{-1}$ and \pm 0.005 lines $\mathrm{mm}^{-1}$, respectively at groove density of $\sim 1740$ lines $\mathrm{mm}^{-1}$ of holographic laser pulse compression gratings. A simple single detector based optical set-up with fixed optical elements to avoid mechanical eccentric errors, if any, due to goniometric movement of a rotatory stage, has been proposed to estimate absolute groove density of gratings. A modified Fizeau or a modified Michelson interferometer based optical set-up has been used to estimate inter-grating groove density errors of gratings. Various gratings from different manufacturers were examined for their absolute groove densities and inter-grating groove density errors.
\end{abstract}

Keywords. Laser pulse compression; diffraction holographic gratings; absolute groove density; inter grating groove density errors.

\section{Introduction}

Laser pulse stretcher [1] and compressor [2] are essential sub-systems of any chirped pulse amplification [3] or optical parametric chirped pulse amplification [4] based ultra-short pulse high power laser systems. These sub-systems often use both angular and non-angular dispersive optical elements like prisms, gratings, grisms (grating on prism surfaces), chirped mirrors and long length optical slabs or optical wave guides exploiting natural or engineered non-angular dispersion in a thinner or longer optical media in both positive and negative dispersion regimes. While angular dispersive elements are widely used to achieve larger temporal stretching or compression, thinner engineered non-angular dispersion is often used for laser pulses with over octave spectral bandwidths. High energy, high power laser systems involving sub 50 fs to sub ps laser pulses mostly use plane reflective gratings for their pulse stretcher and compressor stages. Gratings of suitable groove densities are chosen for a desired laser pulse parameters e.g., pulse duration, pedestal, short range coherent pulse contrast, etc. For a CPA or OPCPA based laser system there may be identical or different groove densities for pulse stretcher and compressor stages [5, 6]. Next, in a practical pulse compressor, one may have gratings with groove density deviated from the designed values

*For correspondence due to various reasons e.g., gratings from two different manufacturers fabricated under different conditions.

Estimating absolute groove densities and inter gratings groove density errors with sub ppm level is desirable both for their characterization and use in a particular application such as laser pulse compression, wherein compressed pulse beam fidelity is eventually dictated by the inter- and intragroove density errors. In most of situations, one rely on values provided by the manufacturer of the gratings. For example, Plymouth grating laboratory, USA fabricate holographic laser pulse compression gratings $[7,8]$ with inter and intra grating groove density errors of 0.001 lines $\mathrm{mm}^{-1}$ (groove spacing error of $0.33 \mathrm{pm}$ at groove spacing of $574712.64 \mathrm{pm}$, i.e., groove density of 1740 lines $\mathrm{mm}^{-1}$ ) over size of around one meter. To estimate groove spacing with sub-nanometer accuracies $[9,10]$ grating surface can be imaged using direct surface imaging techniques such as atomic force microscopy, scanning electron microscopy, etc. and in principle, variation of groove spacing can also be estimated with enhanced precision by scanning a larger section of grating and then performing Fourier transformation to determine spatial modulation frequency and hence groove spacing and errors. However, it is practically difficult to estimate absolute groove spacing and groove spacing errors with desired accuracies over large area of a laser pulse compression grating using above mentioned techniques. In contrast, it is easier to estimate these errors indirectly by measuring the effect, e.g., space-resolved diffraction angle, caused by absolute groove density of the 
grating and its spatial variations. In principle, diffraction angle and its variation over grating size can be easily monitored even without any reference grating and hence absolute groove spacing and its variation can be determined. Further, in the case of laser pulse compression gratings, one may study variation of various pulse parameters after parallel pair of plane gratings, wherein one grating is a referenced one. Next, there are few reports dealing with indirect techniques to estimate absolute groove densities [11], and inter-grating groove density errors [12-14] of laser pulse compression gratings either using referenced calibrated grating or using complicated optical set-up to achieve accuracy of measurement to few ppm level at typical groove densities of around few 1000 lines $\mathrm{mm}^{-1}$. Estimating inter-grating groove density errors with accuracies of 0.001 lines $\mathrm{mm}^{-1}$ (i.e., $\sim 0.6 \mathrm{ppm}$ level at 1740 lines $\mathrm{mm}^{-1}$ ) shall require estimation of differential angle of diffraction of $\sim 0.5 \mu \mathrm{rad}$, which in turn shall require well collimated laser beams and accurate angle measurement detector. While estimating inter-grating groove density errors with mentioned accuracies is relatively a simpler task, determining absolute groove densities with sub ppm level remain a challenging task in standard laboratory conditions.

In this paper, experimental studies are reported on estimation of absolute grating groove density, inter-grating groove density errors with detector limited accuracies below \pm 0.23 lines $\mathrm{mm}^{-1}$ and \pm 0.005 lines $\mathrm{mm}^{-1}$, respectively at grating density of $\sim 1740$ lines $\mathrm{mm}^{-1}$. A simple single detector optical set-up, involving no movable parts thus eliminating mechanical eccentric errors of rotatory stage, if any, generally used in goniometric set-up, has been proposed to estimate absolute groove density. A modified Fizeau or a modified Michelson interferometer based optical set-up has been used for inter-grating groove density errors. Various gratings from different manufacturers were examined for inter- and intra-grating groove density errors using the proposed optical set-up and using a standard commercial $\mathrm{He}-\mathrm{Ne}$ laser operating at wavelength of $632.8 \mathrm{~nm}$.

\section{Principle}

\subsection{Estimation of absolute grating groove density}

In principle, the absolute groove density $(\mathrm{N})$ or groove spacing $\left(\mathrm{d}=\mathrm{N}^{-1}\right)$ of a grating can be estimated by measuring the angle of incidence and diffraction in a standard goniometric set-up using well collimated laser beams. In such a case, accuracy in estimation of groove spacing or groove density is limited to the accuracies in measuring both these angles. Next, measurement of the two angles i.e., angle of incidence $(\alpha)$ and diffraction $(\beta)$ can be avoided by using gratings in Littrow configuration (both angles are equal in this case). This in turn also helps to reduce error in estimation of grating groove density, as there is only one parameter to be measured for a calibrated laser source at specified wavelength $(\lambda)$ chosen for a given grating groove density. Grating groove density $(\mathrm{N})$ is estimated from standard grating equation at Littrow configuration, as

$$
\mathrm{N}=2 \lambda^{-1} \sin \alpha
$$

And error in estimation of groove density $(\Delta \mathrm{N} / \mathrm{N})$ due to error in estimation of angle $\Delta \alpha$ and due to error in wavelength calibration $\Delta \lambda$ is

$$
\frac{|\Delta \mathrm{N}|}{\mathrm{N}}=\cot \alpha|\Delta \alpha|+\frac{|\Delta \lambda|}{\lambda}
$$

From Eq. (2), it can be easily shown that to achieve accuracy of 0.1 lines $\mathrm{mm}^{-1}$ in absolute estimation of grating groove density without any standard reference grating, one would require accuracy of $36 \mathrm{pm}$ in absolute wavelength in a given medium at $632.8 \mathrm{~nm}$ and accuracy of $0.002^{\circ}$ in angle of incidence for a grating groove density of 1740 lines $\mathrm{mm}^{-1}$.

Measuring larger angles with desired accuracies is a little difficult task due to difficulty in intercepting reference and diffracted/reflected beams on to a single detector to achieve single shot measurement. The problem of measuring large angles with high accuracies, can be simplified by transforming larger angles to smaller ones for measurement so as to allow both reference and diffracted/reflected beams on to single detector in a fixed set-up without use of any rotational stage. Such a transformation can be achieved by known beam rotations using appropriate prisms as illustrated in figure 1.

Since prisms can be fabricated with angular errors smaller than few arc second accuracies and therefore measurement of beam angles can also be very accurate. Next, it is desirable to use prisms at minimum deviation to enhance accuracies in absolute angle measurement as errors in positioning prisms is less $\left(\delta \theta_{\mathrm{d}} / \delta \theta_{\text {inc }} \sim 0\right)$ at minimum deviation condition, where $\theta_{\text {inc }}$ is the angle of incidence for the prism. It is easy to understand that type and number of prisms is determined by the expected nearest value of angle of incidence at Littrow configuration for a given grating and laser wavelength. In situations where it is not possible to have all prisms positioned at minimum deviation (e.g., due to their non-availability), one can use right angle prisms to achieve desired beam rotations. The errors in positioning right angle prisms can also be avoided by monitoring respective back reflections.

For a grating with typical groove density of 1740 lines $\mathrm{mm}^{-1}$ at Littrow configuration, angle of incidence and diffraction is calculated to be $33.404^{\circ}$ at specified laser wavelength of $632.8 \mathrm{~nm}$, which shall require beam rotation of $\sim 66^{\circ}$ anticlockwise or $\sim 294^{\circ}$ clockwise to go back towards source to be intercepted using a beam sampler on to a single detector. Three prisms have been used, as shown in figure 1 , to achieve beam rotation of $\sim 90^{\circ}, \sim 180^{\circ}$, and 


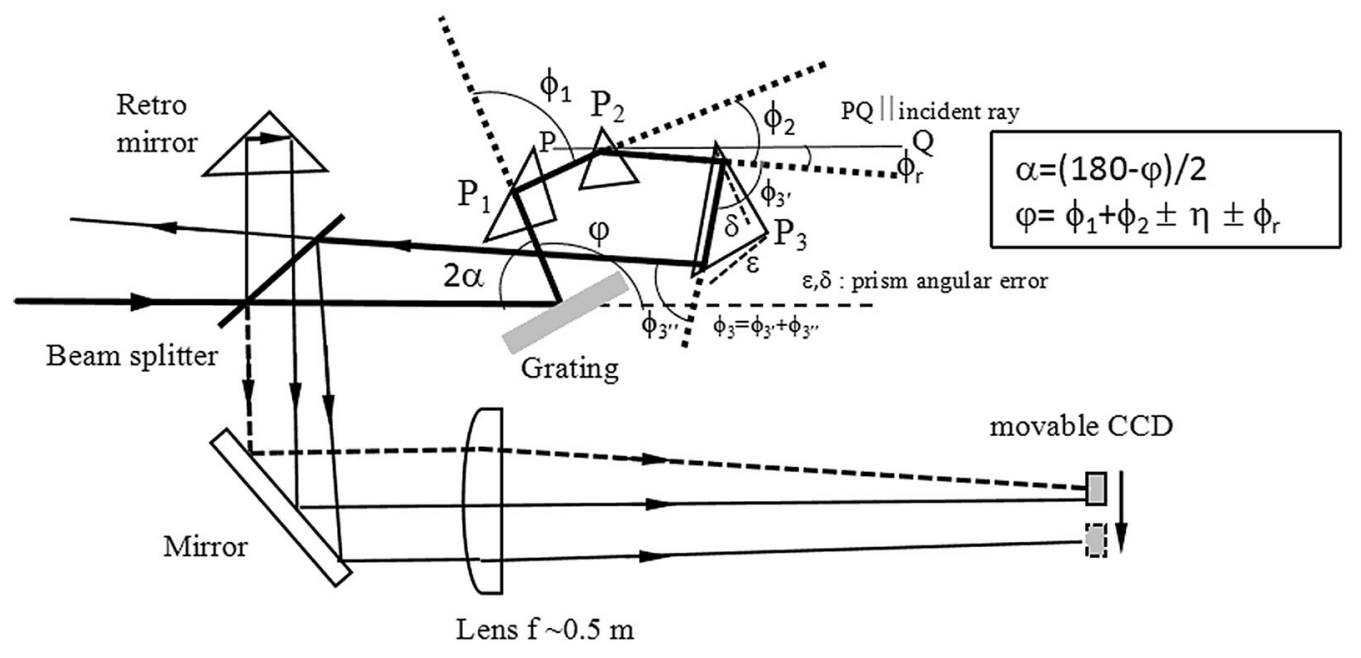

Figure 1. Experimental set-up for estimation of absolute groove density of the gratings.

$\sim 26^{\circ}$ using prism at minimum deviation with apex angle of $\sim 45^{\circ}$.

The angle of incidence $\alpha$, is determined by estimating angle $\varphi$, achieved through measured residual beam angle $\phi_{\mathrm{r}}$, and estimated beam rotations $\phi_{1}, \phi_{2}$, and $\phi_{3}$ provided by the prisms $\mathrm{P}_{1}, \mathrm{P}_{2}$ and $\mathrm{P}_{3}$ from measured prism angular errors $\varepsilon$ and $\delta$ as defined for prism $\mathrm{P}_{3}$ shown in figure 1 , and refractive index of prism material. Angle $\phi_{2}$ is angle of minimum deviation for a prism $\mathrm{P}_{2}$ with apex angle $\mathrm{A}$, and is estimated as $\theta_{d}=2 \sin ^{-1}\{n \sin (0.5 \mathrm{~A})\}-\mathrm{A}$, where $\mathrm{n}$ is the refractive index of the prism material. Any variation in angle of minimum deviation $\theta_{\mathrm{d}}$ with parameters of a prism is calculated as $\delta \theta_{\mathrm{d}} /\left.\delta \mathrm{n}\right|_{\mathrm{A}}=2 \sin (0.5 \mathrm{~A})\left\{1-\mathrm{n}^{2} \sin ^{2}\right.$ $(0.5 \mathrm{~A})\}^{-1 / 2} \quad$ and $\quad \delta \theta_{\mathrm{d}} /\left.\delta A\right|_{\mathrm{n}}=\mathrm{n} \cos (0.5 \mathrm{~A})\left\{1-\mathrm{n}^{2} \sin ^{2}\right.$ $(0.5 \mathrm{~A})\}^{-1 / 2}-1$. Prior to use of prisms, it is desirable to know the angular errors in order to estimate beam angle and hence, absolute grating groove density. For a given right angle prism, angular errors are estimated by recording back reflections using $\mathrm{CCD}$ based angle detector, by keeping prisms for $90^{\circ}$ and $180^{\circ}$ beam rotations, where in beam back reflection errors are expressed as $4 n \delta+2 n \varepsilon$ and $4 n \varepsilon$, respectively. For given angular errors of the prisms, the beam rotations $\phi_{1}$ and $\phi_{3}$ is estimated as $90-2 n \delta-(n+1) \varepsilon$ and $180+2 \mathrm{n} \varepsilon$, respectively in the two situations. Angle of incidence $\alpha$, thus can be determined using relation $\alpha=(180-\varphi) / 2$, where $\varphi$ is the net beam rotation such that rotated beam go back to the direction of laser source and same is estimated from measured angles $\phi_{1}, \phi_{2}, \phi_{3}$ and $\phi_{\mathrm{r}}$, as shown in figure 1 . It is worth mentioning here that error in estimating angle of incidence $\alpha$, by measuring angle $\varphi$, is also reduced to half.

The residual beam angle $\phi_{\mathrm{r}}$, relative to reference incident beam is measured using a focusing lens and CCD camera interfaced to a computer. As mentioned earlier in some cases, availability of suitable prisms may be limited for given grating and laser wavelength, it is highly desirable to have much larger range of angle measurement, which may be enhanced with lens with shorter focal length, and is often trade off with accuracies to allow single shot measurement. One may use CCD coupled with linear translational stage, kept at $90^{\circ}$ to reference beam to reduce cosine error, to allow measurements for both the beams as shown in figure 1. Accuracy and range of residual angle measurement is dictated by the focal length of a focusing lens and CCD pixels, while the accuracies in estimating various beam rotations by right angle prisms and angle of minimum deviation is governed by the accuracies in determining prism apex angles, angular errors, and refractive index of the prism material.

The inter-grating groove density errors can also be estimated using experimental set-up shown in figure 1 by placing two gratings side by side and estimating beam angular errors for the two gratings. However, it may be difficult to estimate absolute grating groove densities with sub ppm level using experimental set-up given in figure 1 due to limited laser beam diameter for given optics size.

\subsection{Estimation of inter-grating groove density errors}

Inter grating groove density errors can be determined easily with sub ppm level accuracies by replacing single grating with two gratings kept side by side and estimating any difference in diffraction angles for the two gratings at constant angle of incidence using well collimated laser beams and a suitable angle detector in a modified Fizeau or a modified Michelson interferometer, as shown in figure 2(I) and 2(II), respectively. Absolute groove density of any grating may also be estimated with sub ppm level accuracies if a standard calibrated reference grating is available. In either experimental set-up, far field beam profiles of reference, reflected, and diffracted beams from 
two gratings and interferogram between reference and diffracted beams from two gratings are recorded using a respective CCD camera, marked as 1,2 , respectively in respective figures to measure differential angle of diffraction $(\Delta \beta)$ used to estimate the inter-grating groove density error $\left(\mathrm{N}_{2}-\mathrm{N}_{1}\right)$ as

$$
\mathrm{N}_{2}-\mathrm{N}_{1}=\left(\lambda^{-1} \cos \beta\right) \Delta \beta
$$

And error in the estimation of inter-grating groove density due to error in estimation of angle $\Delta \beta$ and due to error in wavelength calibration $\Delta \lambda$, and error in estimation of differential angle $\Delta(\delta \beta)$ can be given as

$$
\frac{\left|\Delta\left(\mathrm{N}_{2}-\mathrm{N}_{1}\right)\right|}{\left(\mathrm{N}_{2}-\mathrm{N}_{1}\right)}=\tan \beta|\Delta \beta|+\frac{|\Delta \lambda|}{\lambda}+\frac{|\Delta(\delta \beta)|}{\delta \beta}
$$

In the case of two gratings under examination for inter groove density errors in either of experimental set-up, any difference in the groove density of two gratings for given angle of incidence shall result in different diffraction angles for beams from two gratings for identical angle of incidence. Different angle of diffraction can also occur either due to different angle of incidence or tilt error of the grating assembly or due to any deviation from planar grating substrate. Therefore, it is ensured to have an identical angle of incidence by monitoring reflected far field beam profile from two assumed planar gratings substrate.

Differential angle of diffraction $\left(\Delta \beta=\beta_{1}-\beta_{2}\right)$ is measured at Littrow configuration under condition that differential angle of incidence $\left(\Delta \alpha=\alpha_{1}-\alpha_{2}\right)$ is nearly zero i.e., within few $\mu$ rad to achieve estimation of groove density errors at ppm level. Such an accuracy is achieved by estimating peak locations of the focal spots of the reflected and diffracted beams using slight tip angle between two gratings of tiled grating assembly to avoid piston or tilt like errors in otherwise overlapped focal spots and to achieve accuracies below Rayleigh limited resolution dictated by natural beam divergence. The accuracy in estimation of inter-grating groove density error is primarily dictated by the accuracies in measurement of differential angle of diffraction for given values of angle of diffraction and wavelength. Accuracy in differential angle measurement

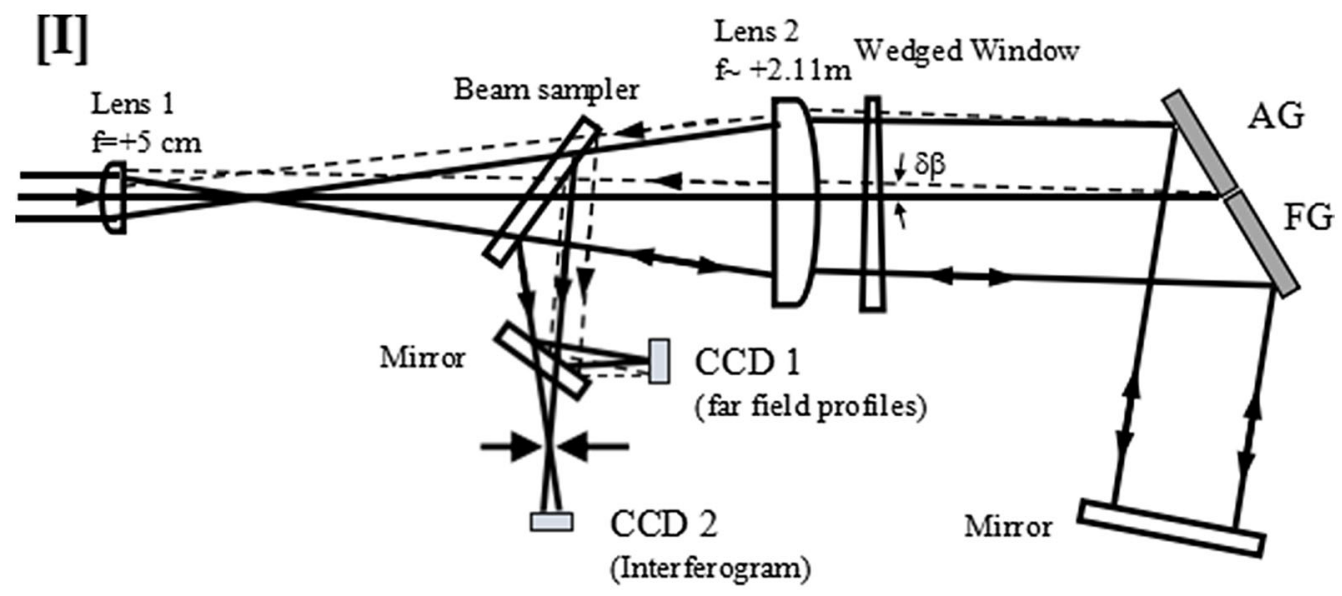

[II]

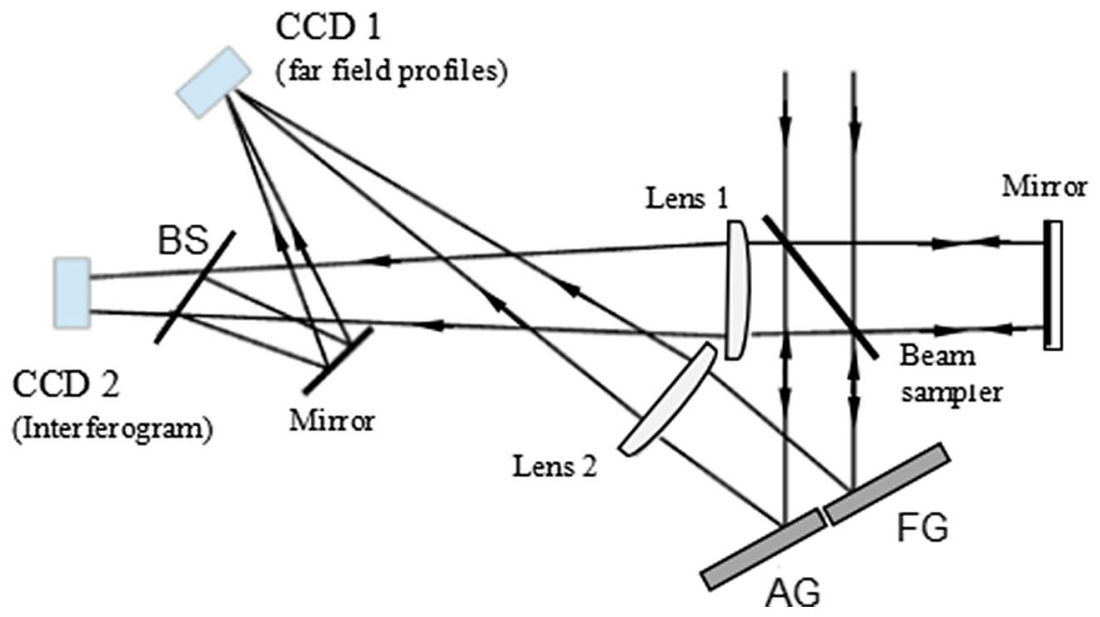

Figure 2. Experimental set-up for estimation of intra-grating and inter-grating groove density errors based on modified (I) Fizeau and (II) Michelson interferometer. 
may be easily achieved below 1 arc second leading to typical accuracy in estimation of $\left(\mathrm{N}_{2}-\mathrm{N}_{1}\right)$ below 0.005 lines $\mathrm{mm}^{-1}$. Thus in principle, using a referenced calibrated grating one may also estimate absolute groove densities with such accuracies. Estimating inter-grating groove density errors with accuracies of 0.001 lines $\mathrm{mm}^{-1}$ shall require estimation of differential angle of diffraction of $\sim 0.5 \mu \mathrm{rad}$, which in turn shall require well collimated laser beams and high precision angle measurement detector with suitable laboratory environment.

\section{Experimental results}

Various gratings from two different manufacturers (Grating\#1: size $6 \times 4 \mathrm{~cm}^{2}$, Grating\#2: size $11 \times 8 \mathrm{~cm}^{2}$, Grating\#3 and \#4: each size $14 \times 12 \mathrm{~cm}^{2}$ ) with nominal groove densities of 1740 lines $\mathrm{mm}^{-1}$ have been examined for absolute groove density errors using experimental set-up shown in figure 1 and one against another in differential groove density measurement using experimental set-up given in figure 2.

Prior to use of prisms for beam rotation as illustrated in figure 1, these were examined for any angular errors in order to estimate correct rotation of beam. In the present example, the prism angular errors $(\varepsilon, \delta)$ are estimated to be $\left(-0.001^{\circ}, 0.011^{\circ}\right),\left(-0.017^{\circ}, 0.021^{\circ}\right)$ and $\left(-0.006^{\circ}\right.$, $0.018^{\circ}$ ) for prisms $\mathrm{P}_{1}, \mathrm{P}_{2}$ and $\mathrm{P}_{3}$, respectively with typical accuracies of $\pm 0.0002^{\circ}$. From these values, beam rotation is estimated to be $89.971^{\circ}, 179.983^{\circ}$ for prisms $P_{1}$ and $P_{3}$, respectively with typical accuracies of $\pm 0.0005^{\circ}$, respectively. Value of beam rotation due to prism $\mathrm{P}_{2}$ kept at minimum deviation is estimated to be $25.889^{\circ}$, and $25.870^{\circ}$ for measured prism apex angles of $45.021^{\circ}$ and $44.995^{\circ}$, respectively and refractive index of prism material (BK7) of 1.5151 at wavelength of $632.8 \mathrm{~nm}$ [15]. Residual beam angle is measured, in two experimental situation, to be $2.725^{\circ}$ and $2.685^{\circ}$ for apex angle of prism $\mathrm{P}_{2}$ of $45.021^{\circ}$ and $44.995^{\circ}$, respectively. From these two measurements carried out independently at two apex angles of prism $\mathrm{P}_{2}$, groove density of grating\#1 is estimated to be 1740.06 lines $\mathrm{mm}^{-1}$ using Eq. (1). Error in estimation of angle, $\alpha$, due to error in estimated beam rotations and measured residual beam angle, and error in placing various prisms, shall lead to error in groove density and is estimated to be \pm 0.23 lines $\mathrm{mm}^{-1}$. Likewise, averaged groove density of grating \#2, \#3 and \#4 is estimated to be 1740.06 lines $\mathrm{mm}^{-1}, 1744.29$ lines $\mathrm{mm}^{-1}, 1744.75$ lines $\mathrm{mm}^{-1}$, respectively.

While experimental set-up given in figure 1 may be used for inter-grating groove density measurement with limited accuracies due to beam or optics size limitations, experimental set-up shown in figure 2 has been used independently for such measurements with enhanced accuracies. A well collimated laser beam of $\sim 70 \mathrm{~mm}$ diameter with beam natural divergence of $\sim 11 \mu \mathrm{rad}$, tested using a wedge plate causing shearing interferogram, was used to illuminate tiled grating assembly shown in figure 2 to study inter-grating groove density errors. The CCD camera of far field profile monitor was placed at best focal condition for both the reflected and diffracted beams. Inter-grating groove density errors, between gratings \#1 and \#2, gratings $\# 3$ and \#4, and grating \#2 and \#3 is estimated to be around 0.03 lines $\mathrm{mm}^{-1}, 0.41$ lines $\mathrm{mm}^{-1}$, and 3.58 lines $\mathrm{mm}^{-1}$, respectively with typical detector limited accuracy below \pm 0.005 lines $\mathrm{mm}^{-1}(\sim 3 \mathrm{ppm}$ at groove density of 1740 lines $\mathrm{mm}^{-1}$ ). It may be noted from absolute measurements on grating groove density that differential groove density of grating \#2 and \#3 is observed to be larger than measured value of inter-grating groove density errors using set-up shown in figure 2(I) . The difference between two values is attributed to possible refraction errors and shift in wavelength of the laser beam. The peak locations of four far field beam profiles are estimated by taking average of centroids at multiple level intensity thresholds to enhance the accuracies below 1 pixel of CCD. However, the repeat-ability in the measurements carried out at different time intervals was observed to be larger than expected detector limited values and is attributed to shifts in the either focal spots due to inherent or induced beam pointing errors caused by mechanical vibrations disturbing various mounts housing optical components of the optical set-up. To ensure this variation of inter-grating groove density errors was recorded over short and long term basis at different time intervals at different focusing conditions. Typical variations on inter-grating groove density errors recorded using Fizeau interferometer based optical set-up are shown in figure 3(II) for two conditions, namely, enhanced and reduced mechanical vibrations leading to errors in angle measurement. Estimation of inter-grating groove density errors may also be affected by the detector position around focus, as illustrated in figure 3(II)) using measurements carried out in step of $5 \mathrm{~mm}$ around focus with total scan distance of 10 $\mathrm{mm}$ in either direction. Any deviation of $\Delta \alpha$ from minimum achievable value shall cause error in estimation of intergrating groove density due to the fact that error in measurement $\Delta \alpha$ due to back reflection is not accounted in the present case. Therefore, value of $\Delta \alpha$ in each step shown in figure 3(II) was minimized below $1 \mu \mathrm{rad}$ using an electronic nanometric actuator after the detector is positioned at appropriate regions marked as IIa, IIb and IIc in figure 3. Further, it may also be seen from figure 3(II) that errors in estimation is also minimized at focus due to reduced errors in estimation of peak location of respective focal spots as threshold condition remain unchanged during the each measurement. These results indirectly indicate repeatability and reliability in the estimation of the inter-grating groove density errors caused by beam and tiled grating assembly, which can be greatly improved by using stable mounts for TGA. From figure 3(II), reliability in the estimation of inter-groove density is observed to be below 0.02 lines $\mathrm{mm}^{-1}$ and 0.01 lines $\mathrm{mm}^{-1}$ at focus for enhanced and 
[I]

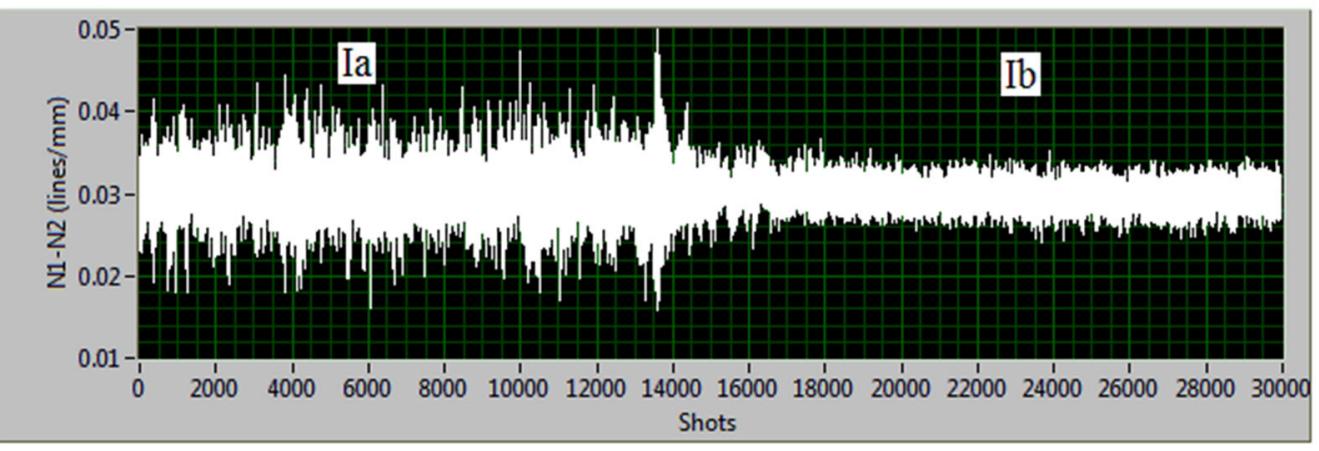

[II]

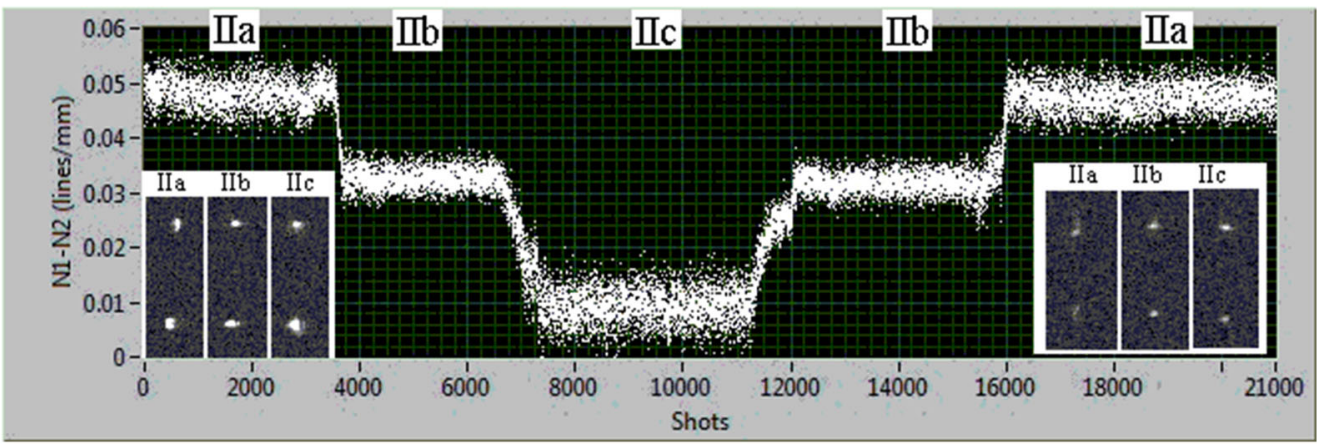

Figure 3. Typical variation of inter-grating groove density: (I) monitored with enhanced (a) and reduced vibrations (b) by switching laboratory air conditioners on and off, respectively; and (II) monitored by placing detector at $5 \mathrm{~mm}$ before focus (a), at focus (b) and at 5 $\mathrm{mm}$ after focus (c) in both forward and backward scan. Typical profiles of diffracted and reflected beams from TGA corresponding to IIa, IIb and IIc are also shown in left and right inset of figure II, respectively.

reduced mechanical vibrations obtained by switching laboratory air conditioners on and off respectively, while the repeatability was observed to be within 0.02 lines $\mathrm{mm}^{-1}$. Intra-grating groove density error, achieved by blocking central portion of expanded beam in set-up shown in figure 2(II), was observed to be below measurement limit of the present set-up. Next, it is important to mention here that differential averaged groove densities of the two gratings, are estimated due to space averaged measurements in a single Shack-Hartmann detector [16]. Analysis of spaceresolved differential measurement of wave front errors in diffracted and reflected beams from grating is desirable to obtain localized variation in grating groove density.

Typical far field profiles of reflected and diffracted beam are recorded using CCD based far field profile monitor and given in figure 4. Figure 4(II) depicts clearly resolved far field profiles of reflected and diffracted beams from two gratings using tip angle of tiled grating assembly in order to estimate peak locations of each profile and hence differential angle of incidence $\Delta \alpha$ and diffraction $\Delta \beta$. Since different lobes are clearly resolved, their peak location can be easily estimated with sub-pixel accuracies and hence

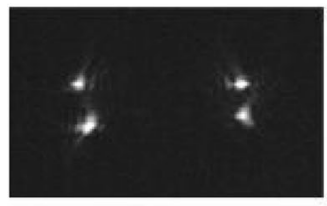

(I)

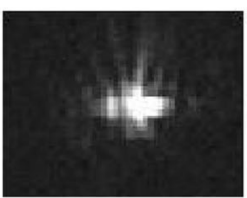

(IIa)

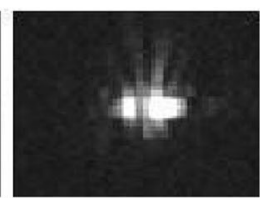

(IIb)

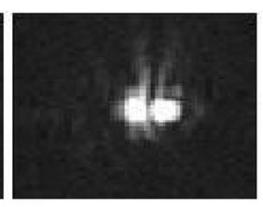

(IIc)

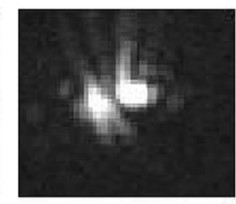

(IId)

Figure 4. Typical far field profiles of reflected and diffracted beams (I) from two grating assembly, and overlapped diffracted beam profiles with different values of piston, tilt, or groove density errors without tip error (IIa to IIc) and with small amount of tip error (IId). Sub-figures shown in 4(I) and 4(II) are not to the scale. Different far field beam profiles shown in 4(I) are vertically separated using tip angle of tiled grating assembly in order to estimate their peak locations for differential angle measurement below Rayleigh limited resolution. 
determination of differential beam pointing below diffraction limited resolution. Figure 4(IIa) to figure 4(IId) show typical overlapped far field profile of diffracted beams from tiled grating assembly with different values of piston, tilt, or groove density errors without (IIa to IIc) and with (IId) small amount of tip error. Far field beam profiles, shown in figure 4(II), clearly illustrate that it is difficult to estimate differential angle of diffraction due to simultaneous presence of piston, tilt and groove density errors resulting in complicated far field beam profile and hence a detailed analysis of overlapped far field profile is desirable to obtain differential angle of diffraction.

\section{Conclusion}

In conclusion, a simple single detector based optical set-up, with fixed optical elements to avoid eccentric errors if any due to mechanical goniometric movements, has been proposed to estimate absolute groove density. A modified Fizeau or a modified Michelson interferometer based optical set-up was used to estimate inter-grating groove density errors. Experimental studies are reported on estimation of absolute groove density, inter-grating groove density errors in a various gratings from different manufacturer with detector limited accuracies below \pm 0.23 lines $\mathrm{mm}^{-1}$ and \pm 0.005 lines $\mathrm{mm}^{-1}$, respectively in the present set-up. Absolute groove density with above mentioned accuracies can also be estimated using a standard calibrated reference grating. Estimating inter-grating groove density errors with accuracies better than 0.001 lines $\mathrm{mm}^{-1}$ $\left(\sim 0.5 \mathrm{ppm}\right.$ at 1740 lines $\left.\mathrm{mm}^{-1}\right)$ shall require estimation of differential angle of diffraction of $\sim 0.5 \mu \mathrm{rad}$, which in turn shall require high quality incident wave front involving use of spatial filters, optics and much higher resolution of the angle detector with differential wave fronts measurement capability in reflected and diffracted laser beams.

\section{Acknowledgements}

AKS thanks Shri D Daiya and Ms J Sharma from High Energy Laser Development Laboratory, Advanced Lasers and Optics Division of RRCAT, Indore for their participation in initial experiment on estimating inter-grating groove density errors.

\section{References}

[1] Martinez O E 19873000 times grating compressor with positive group velocity dispersion: Application to fiber compensation in 1.3-1.6 micron region. IEEE J. Quantum Electron. 23: 59-64

[2] Treacy E B 1969 Optical pulse compression with diffraction gratings. IEEE J. Quantum Electron. 5: 454-458

[3] Strickland D and Mourou G 1985 Compression of amplified chirped optical pulses. Opt. Commun. 56: 219-221

[4] Dubietis A, Jonusauskas G and Piskarskas A 1992 Powerful femtosecond pulse generation by chirped and stretched pulse parametric amplification in BBO crystal. Opt. Commun. 88(4-6): 437-440

[5] Bagnoud V and Salin F 1998 Global optimization of pulse compression in chirped pulse amplification. IEEE J. Sel. Top. Quantum Electron. 4: 445-448

[6] Squier J, Barty C P J, Salin F, Blanc C L and Kane S 1998 Use of mismatch grating-pairs in laser pulse compression systems. Appl. Opt. 37(9): 1638-1641

[7] Jitsuno T, Motokoshi S, Okamoto T, Mikami T, Smith D, Schattenburg M L, Kitamura H, Matsuo H, Kawasaki T, Kondo K, Shiraga H, Nakata Y, Habara H, Tsubakimoto K, Kodama R, Tanaka K A, Miyanaga N and Mima K 2008 Development of $91 \mathrm{~cm}$ size grating and mirrors for LEFX laser system. J. Phys: Conf. Ser. 112: 032002,1-4

[8] www.plymouthgratings.com (reference is to address issues of laser pulse compression gratings; authors do not conform quality of gratings)

[9] Boyd R D, Britten J A, Decker D E, Shore B W, Stuart B C, Perry M D and Li L 1995 High efficiency metallic diffraction gratings for laser applications. Appl. Opt. 34(10): 1697-1706

[10] Britten J A, Perry M D, Shore B W and Boyd R D 1996 Universal grating design for for pulse stretching and compression in the 800-1100-nm range. Opt. Lett. 21(7): $540-542$

[11] Yoon T H, Eom C I, Chung M S and Kong H J 1999 Diffractometric methods for absolute measurement of diffraction grating spacing. Opt. Lett. 24(2): 107-109

[12] Guo C and Zeng L 2008 Measurement of period difference in grating pair based on compensation analysis of phase difference between diffraction beams. Appl. Opt. 48(9): $1651-1657$

[13] Lim J and Rah S 2004 Technique of measuring the groove density of a diffraction grating with elimination of eccentric effect. Rev. Sci. Instrum. 75: 780-782

[14] Wang Q, Liu Z, Chen H, Wang Y, Jiang X and Fu S 2015 The method for measuring the groove density of variableline-space gratings with elimination of the eccentricity effect. Rev. Sci. Instrum. 86: 023109-4

[15] https://refractiveindex.info/?shelf=glass\&book=BK7\&page= SCHOTT

[16] Zou W, Thompson K P and Rolland J P 2008 Differential Shack-Hartmann curvature sensor: local principal curvature measurements. J. Opt. Soc. Am. A 25(9): 2331-2337 